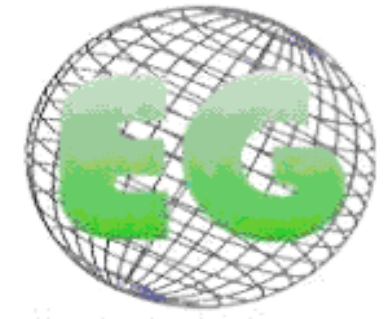

ISSN 1695-6141 N 27
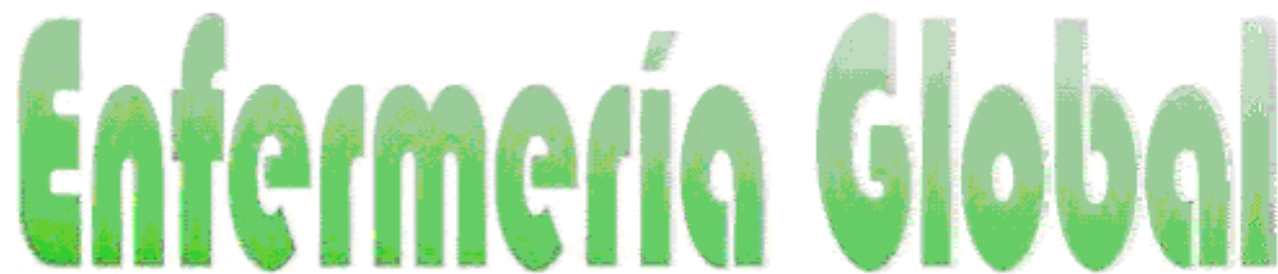

Revista electrónica trimestral de Enfermería

Julio 2012

www.um.es/egloball

REVISIONES

\title{
Relevancia de instrumentos de recolecta de datos en la calidad del cuidado
}

Relevance of data collection instruments for quality of care

*Guilherme, C., " Carvalho, EC de, ***Landeros López, M.

\begin{abstract}
*Lcda. en Enfermería. Bolsista de Apoyo técnico CNPq del Laboratorio de Enfermería y Comunicación. Escuela de Enfermería de Ribeirão Preto. Universidad de São Paulo, Brasil. E-mail: enfcom@eerp.usp.br **Doctora en Enfermería. Profesora Titular de la Escuela de Escola de Enfermería de Ribeirão Preto. Universidad de São Paulo. *** Doctora en Enfermería. Profesora Investigadora de la Facultad de Enfermería de la Universidad Autónoma de San Luis Potosí. México.
\end{abstract}

Palabras clave: instrumentos; validación; recolección de datos; enfermería.

Keywords: instruments; validation; data collection; nursing

\section{RESUMEN}

Considerando la importancia del proceso enfermero en la práctica basada en evidencias, buscamos identificar instrumentos validados y confiables para la recolección de datos en pacientes. Realizamos una revisión integrativa utilizando las bases CINAHL, BVS, PUBMED. Fueron incluidos artículos disponibles a texto completo en inglés, español y portugués, se excluyeron las revisiones que no contenían descritos los pasos metodológicos y los que no abordaban el tema. En los 40 estudios analizados fueron identificados instrumentos aplicables a diferentes áreas como geriatría y gerontología, terapia intensiva, oncología, psiquiatría, nutrición, infectología, salud del trabajador, cuidados paliativos, cardiología, entre otros. La mayoría de los instrumentos $47.5 \%$ aplicaba para población anciana. Enfermería al utilizar instrumentos validados en su práctica pasa a ejercer una asistencia con mayor calidad. Sin embargo, es evidente que diversos comportamientos y manifestaciones clínicas todavía no disponen de instrumentos validados para su evaluación.

\section{ABSTRACT}

In view of the importance of the nursing process in evidence-based practice, we attempt to identify validated and reliable instruments to collect data from patients. An integrative review was carried out in the databases CINAHL, BVS and PUBMED, using the words "nursing" and "validated clinical assessment tools", as well as "validation studies", "reproducibility of results" and "nursing assessment". Full articles available in English, Spanish or Portuguese were included, excluding reviews that did not follow the methodological steps described and did not address the theme. In the 40 analyzed studies, instruments were identified that are applicable to different areas, such as geriatrics and gerontology, intensive care, oncology, psychiatry, nutrition, infectology, occupational health, palliative care, cardiology, among others. Instruments were found to evaluate the following areas: risk of falls, patient satisfaction, quality of life, sleep, depression, mental confusion, dementia, pain, 
behavior, nutritional status, violence, personality, psychosis, breastfeeding, nausea and vomit, fatigue, end of life needs, sedation and agitation, abstinence from opioid use, pressure ulcers, capacity of workers with musculoskeletal problems to restart activities, immunological status of patients with HIVIAIDS, and patients' degree of knowledge about their disease. There was an increase in the number of publications from 1990 to 2006. Most of the instruments, $47.5 \%$, were applicable to elderly populations. Nursing, when using validated instruments in its practice, provides more qualified care. Several behaviors and clinical manifestations exist, however, without validated instruments for their assessment.

\section{INTRODUCCIÓN}

Para el fortalecimiento de la autonomía del enfermero ${ }^{1-2}$ la intuición viene siendo sustituida en la práctica profesional por la inclusión de resultados de investigaciones que dan soporte a la evaluación de las condiciones de salud del paciente ${ }^{3}$.

En el desarrollo del proceso de enfermería, es relevante obtener datos que permitan la identificación de los fenómenos o los diagnósticos presentes que constituirán el marco de referencia para la elaboración del plan y la implementación de las acciones de asistencia.

Se entiende, por lo tanto, la relevancia de instrumentos validados, sin embargo, todavía son pocos considerando todo lo que abarca la profesión, y los que están validados no siempre son conocidos y utilizados. Así, tenemos como objetivo en este trabajo identificar instrumentos validados y confiables que puedan ser aplicados en la fase de recolección de datos del proceso de Enfermería.

\section{METOdOLOGÍA}

Realizamos una revisión integrativa ${ }^{4}$; la búsqueda fue llevada a cabo en las bases CINAHL, BVS y PUBMED utilizando las palabras clave "nursing" y "validated clinical assessment"; así también con "validation studies", "reproducibility of results" y "nursing assessment". Los títulos y los resúmenes fueron analizados; aquellos que no presentaban claramente la construcción o validación de los instrumentos fueron descartados. De los 132 resúmenes identificados 12 fueron excluidos por mostrar una revisión bibliográfica sin criterios de selección de los artículos; 46 no se adecuaron a los objetivos del trabajo ya que correspondían a evaluaciones del servicio de enfermería, de instrumentos médicos o validación de diagnósticos; otros, 17 por estar repetidos, es decir, estaban en más de una base de datos, y 1 por no estar disponible en idioma inglés, portugués o español. A continuación, de los 56 artículos restantes fueron excluidos 16 por no encontrarse disponibles on-line, finalmente el total fue de 40 artículos.

\section{RESULTADOS}

\section{Caracterización de los artículos examinados}

Los 40 artículos $^{5-44}$ fueron analizados primeramente en relación a las características generales de identificación, divulgación, tipo de estudio y sujetos involucrados.

En cuanto a la fecha de publicación, las investigaciones fueron divulgadas en el periodo de 1990 a 2009 , presentando un mayor número a partir de 1999, destacando los años de $2004^{7,16,29,41,42,44}, 2006^{6,8,11,30,38,39}$ y $2008^{21,26-28,37,43}$.

Al identificarse la población de los estudios, fue evidenciado que un número importante de estudios $(n=19)$ contenían instrumentos aplicados a la población 
anciana $5,8,10,13,15,16,18,19,21,23,28-30,32,34,36,39,40,43$, seguidos de aquellos que presentaron instrumentos enfocados a los adultos $(n=9)^{6-7,17,22,24,26,35,38,44}$, pacientes pediátricos $(n=2)^{11,25}$, neonatos $(n=2)^{27,41}$, sujetos de diferentes edades $(n=4)^{20,33,37,42}$, y otros que no especificaron la población de estudio $(n=4)^{9,12,14,31}$.

En cuanto a las áreas clínicas, 18 investigaciones estaban dirigidas a Geriatría y Gerontología $^{5,8,10,13,15-16,18-19,21,23,28-30,32,34,39-40,43 ~}, 5$ al área de Terapia intensiva ${ }^{11,17,25,37,41} ; 4$ a Oncología $26,35,38,42$, además de los de Salud del trabajador' ${ }^{9}$ Cuidados Paliativos ${ }^{31}$, Cardiología $^{14}$, y otros 2 que no especificaron ${ }^{12,36}$.

\section{Instrumentos de recolección de datos de pacientes según área clínica}

Según las áreas clínicas fueron identificados instrumentos con distintos propósitos, los instrumentos aplicados en el área de Geriatría y Gerontología estaban enfocados a la identificación y evaluación del riesgo de caída ${ }^{5,21,29-30}$; evaluación de la satisfacción del paciente ${ }^{8}$; evaluación de la calidad de vida percibida por el paciente ${ }^{13}$; evaluación del sueño ${ }^{15}$; evaluación e identificación de depresión ${ }^{16,18,28}$; evaluación de confusión mental ${ }^{10}$; instrumento de identificación de demencia en ancianos ${ }^{32}$; Instrumento para evaluar el dolor en pacientes ancianos con demencia ${ }^{40}$; identificar cambios de comportamiento en pacientes ancianos con demencia ${ }^{39}$; evaluación de las dificultades para los ancianos con demencia para alimentarse ${ }^{19}$; evaluación nutricional de ancianos hospitalizados ${ }^{34}$; evaluación de la morbilidad física ${ }^{36}$; y evaluación de incomodidad en pacientes con Alzheimer ${ }^{43}$.

Los instrumentos citados en los estudios del área de Psiquiatría procuraban: evaluación de los aspectos de la personalidad ${ }^{6}$; instrumentos específicos para precisión de violencia en pacientes psiquiátricos ${ }^{7,44}$; y para evaluación del paciente con psicosis ${ }^{20}$.

Los instrumentos específicos de nutrición trataron sobre la evaluación del amamantamiento en neonatos ${ }^{27}$ y de la evaluación del apetito ${ }^{33}$. Para el área de Oncología presentaron un instrumento de evaluación de náusea y vómito inducidos por la quimioterapia ${ }^{26}$; evaluación e identificación de depresión ${ }^{35}$; y evaluación del sueño ${ }^{42}$. En el área de Cuidados Paliativos fue empleado un instrumento para identificar las necesidades al final de la vida ${ }^{31}$.

En Terapia Intensiva fueron usados instrumentos para evaluación de los niveles de sedación y agitación en pacientes pediátricos ${ }^{11}$; evaluación de confusión ${ }^{17}$; instrumento que evalúa el síndrome de abstinencia a opioides y benzodiazepínicos en UTI pediátrica ${ }^{25}$; prevención del riesgo de úlceras de presión ${ }^{37}$; y evaluación del dolor en neonatos ${ }^{41}$. En el área de salud del trabajador el propósito fue evaluar la capacidad del trabajador con problemas músculoesqueléticos para desempeñar su actividad al regresar al trabajo ${ }^{9}$. Dentro del área de Infectología el instrumento identificado evaluaba el estado inmunológico del paciente con HIV/AIDS ${ }^{22,24}$ y en Cardiología evaluaba el grado de conocimiento del paciente sobre su enfermedad $^{14}$. Otro apuntaba a la identificación y evaluación del riesgo de caída en pacientes hospitalizados sin especificar la edad ${ }^{12}$.

\section{Instrumentos de recolección de datos según validación presentada}

Entre los instrumentos de evaluación del riesgo de caída en ancianos, el instrumento "Morse Falls Scale" y el "STRATIFY" se presentaron comparables al juicio clínico de los enfermeros mostrando precisión ${ }^{5}$. El uso del instrumento "Hendrich Fall Risk Model" para evaluación del riesgo de caída puede predecir casi el $75 \% \%$ de las caídas con $74.9 \%$ de sensibilidad y $73 \%$ de especificidad, siendo validado y probado en ambiente de cuidados agudos ${ }^{12}$. 
Investigadores en Australia hicieron una evaluación del riesgo de caída a través de dos instrumentos, uno específico para evaluar el riesgo de caída en pacientes ancianos que tenían condiciones para permanecer solos, y otro instrumento específico para evaluar pacientes que necesitaban de ayuda para el auto-cuidado. Se identificó que los pacientes que tienen habilidad para estar solos y tienen dos o tres factores de riesgo para caída (ejemplo caídas anteriores, estar internado en una institución, presencia de incontinencia urinaria) tienen tres veces más proporción de caída (sensibilidad $73 \%$, especificidad $55 \%$ ), en cuanto a los pacientes que necesitaban de apoyo para el auto-cuidado y no tenían condiciones para permanecer solos, que tenían de uno a tres factores de riesgo, tuvieron dos veces más la proporción de caída (sensibilidad $87 \%$, especificidad $29 \%)^{21}$.

En Estados Unidos de Norteamérica fue realizada una evaluación de los tipos de instrumentos post-caída existentes en instituciones de cuidado privadas y públicas, encontrándose que la mayoría de las instituciones usaban instrumentos para evaluación del riesgo de caída en lugar de instrumentos de evaluación post-caída (63.7\%), expusieron la importancia de esta distinción pues los instrumentos de evaluación post-caída son específicos para identificar la etiología de las caídas, y señalaron que ningún instrumento validado y probado empíricamente para la evaluación post-caída fue identificado ${ }^{29}$. En un estudio más reciente ${ }^{30}$ fue evaluada la viabilidad del instrumento de evaluación post-caída "Post Fall Index" para instituciones de cuidado, fue obtenida una buena concordancia entre los observadores (70-100\%), se trata de un instrumento validado, confiable y de aplicabilidad clínica para la prevención de caídas.

Para colaborar con la evaluación del comportamiento y de lesiones en las áreas de la memoria, actividades diarias, humor, comportamiento social y disturbio de comportamiento, el "Nurses" Observation Scale for Geriatric Patients" (NOSGER) fue otro instrumento validado para población anciana (Spearman's $\geq 80$ ), puede ser aplicado por enfermeros 0 cuidadores, el nivel de aprobación fue considerado bueno por familiares y cuidadores ${ }^{23}$.

La identificación de demencia en ancianos también fue motivo de investigaciones, el instrumento "Dementia Screening Scale" (DSS) obtuvo una precisión de AUC=0.912 $(95 \% \mathrm{CI}$ 0.89-0.94) alcanzó una mejor validez que la evaluación diagnóstica realizada por el equipo, considerada de fácil aplicación, económica y asociada a bajos niveles de ausencia de respuesta ${ }^{32}$. Los cambios de comportamiento en pacientes ancianos con demencia también pudieron ser medidos por el instrumento "Behavioural and psychological symptoms of dementia" (BPSD) que obtuvo una buena consistencia interna ( $\alpha=0.86)$, concordancia $(\mathrm{ICC}=0.79)$, y test-retest de confiabilidad $(\mathrm{ICC}=0.98)$; los cambios de comportamiento fueron asociados al género masculino, compromiso cognitivo, incapacidad funcional, síntomas neuropsiquiátricos e a elevados niveles de sobrecarga de trabajo en los cuidadores ${ }^{39}$.

Para la evaluación del dolor, cuatro instrumentos fueron correlacionados con la "Observational Rating Scale" para su validación, entre ellas la "Escala Horizontal Visual Analógica" (HVA), "Escala Vertical Visual Analógica" (VVAS), la "Faces Pain Scale" (FPS), y la "6-point verbal reating scale" (VRS), obteniéndose elevada confiabilidad y una correlación fuerte (Spearman's=0.81-0.95; $p<0.01$ ); en tanto que la "Observational Rating Scale" obtuvo moderada correlación con la auto-evaluación por el paciente, tendía a subestimar la intensidad del dolor, y es indicada solo para aquellos pacientes que comprobaran no poder responder a la autoevaluación ${ }^{41}$.

El riesgo de úlceras por presión fue evaluado por el instrumento "Suriadi and Sanda Scale" (SS Scale), aplicado a pacientes internados en Unidades de Terapia intensiva, obtuvo $81 \%$ de sensibilidad, $83 \%$ de especificidad, valor predictivo positivo de $65 \%$, y valor predictivo 
negativo de $91 \%$, considerándose necesario realizar nuevos estudios sobre la validez predictiva posterior al uso de la escala y determinar el punto de corte del score en diferentes poblaciones $^{37}$.

La calidad de vida percibida por los pacientes de una comunidad de ancianos fue evaluada a través de los 12 ítems del instrumento "Assessment of Quality of Life" (AqoL), su validez psicométrica fue considerada aceptable (confiabilidad $\alpha=0.73$ ). Mostró sensibilidad para la previsión de futuros costos en salud, demostró que elevados escores resultan en menos costos futuros a diferencia de los bajos scores ${ }^{13}$.

El instrumento de evaluación de la satisfacción de los pacientes "Satisfaction with the Nursing Home" fue aplicado a los ancianos que viven en instituciones de cuidados con el intento de evaluar la satisfacción con la calidad de los servicios de enfermería, el uso está indicado para investigadores, gobernantes, administradores y profesionales que se dedican a la mejora de la calidad del cuidado de los ancianos institucionalizados ${ }^{8}$.

El conocimiento de pacientes con trastorno cardiaco sobre su propia enfermedad fue medido por el instrumento "Patient Knowledge questionnaire" (PKQ). Por medio de este instrumento la evaluación del conocimiento del paciente se tornó indispensable para identificar las dudas y clarificarlas, además de contribuir a modificar los comportamientos ${ }^{14}$.

El patrón de sueño en ancianos fue medido por el "Observacional Sleep Assessment Instrument" (OSAI), la concordancia fue de $92.7 \%$, se encontró que la mayoría de las variables se mostraron consistentes y correlacionadas, muy similares a las informaciones obtenidas en el monitor portátil, sin embargo, la muestra del estudio fue considerada pequeña por lo que es necesario realizar más estudios en poblaciones mayores ${ }^{15}$. La evaluación del sueño en pacientes con cáncer puede ser evaluada por el "Pittsburg Sleep Quality Index" (PSQI) considerado como relativamente fácil de aplicar, mostró confiabilidad y validez de constructo en la evaluación psicométrica, mas también fueron sugeridas investigaciones adicionales para evaluar confiabilidad, sensibilidad y validación de constructo en pacientes con cáncer de diversas etnias para pasar el uso en estudios clínicos ${ }^{42}$.

De los instrumentos para evaluar la capacidad funcional del trabajador con problemas músculoesqueléticos para desempeñar su actividad al regresar al trabajo, la "Functional Abilities Confidence Scale" (FACS) y el "Occupational Role Performance Questionnaire" (ORQ) tuvieron los mayores porcentajes de evidencias psicométricas, este tipo de instrumento ayuda al acompañamiento en la rehabilitación y del progreso de daños de los trabajadores, ofrece una evaluación comprensible del impacto del tratamiento y su efecto global, e indica la aptitud del trabajador al regresar a su función; otros usos potenciales para estos instrumentos son la evaluación del cambio en la limitación del trabajador, identificar trabajadores con menor producción, reorganizar objetivos y responsabilidades de trabajo disminuyendo la demanda de tareas, además de identificar personas que están en riesgo de daños 9 .

Los signos y síntomas comunes en pacientes con HIV fue el objeto del "Sign and Symptom Check-List for Persons with HIV Disease" (SSC-HIV); los síntomas evaluados fueron: fiebre, fatiga, confusión, náusea y vómito, angustia psicológica, disnea, incomodidad gastrointestinal y diarrea, pudiendo ayudar en el plan de intervención. Como limitación fue puntualizada la carencia de ítems que expresaran la experiencia de las mujeres al vivir con HIV/AIDS. EI manejo de signos y síntomas es considerado el mayor componente del cuidado al paciente con HIV/AIDS, su evaluación en este proceso puede mejorar la comunicación 
entre paciente y profesional, además de facilitar la intervención y diagnóstico precoz ${ }^{22}$. Otro estudio también evaluó los cambios en el estado inmunológico de pacientes con HIV a través del "HIV-Qualiity Audit Marker" (HIV-QAM), su validez predictiva de mortalidad varió de 3 a 6 meses después de iniciado el tratamiento hospitalario para Neumonía relacionada a Pneumocystis carinii ${ }^{24}$.

Los instrumentos que evaluaron el síndrome de abstinencia por opioides dirigen la atención al infante en cuidados de Terapia Intensiva. Se resaltó que tal evento puede afectar al $20 \%$ de los niños expuestos al uso de opioides, y está relacionada con la duración de la infusión de dosis total ${ }^{25}$. El síndrome puede ser evaluado por instrumentos como el "NAS", su uso presentó mayor efectividad que el juicio clínico usado por la mayoría de los centros de neonatología; el "OBWS" mostró fuerte correlación con el juicio clínico de los enfermeros, con una confiabilidad de $80 \%$, especificidad de $87 \%$, y sensibilidad de 50\%; el "Seven Signs of Behavioural Distress" presentó confiabilidad de $95 \%$ entre dos observadores; y el "Sedation Withdrawal Score" no presentó datos validados para sustentar su uso ${ }^{25}$. Se realizó un estudio piloto para validar la escala "State Behavioural Scale" (SBS) con la finalidad de medir los niveles de sedación y agitación en lactantes y niños pequeños intubados, con correlación entre los observadores considerada de moderada a elevada ${ }^{11}$.

Medir la intensidad y duración de la náusea y vómito inducidos por la quimioterapia es benéfico en el control del uso de antieméticos, con este objetivo fueron empleados los instrumentos "MAT" e "INVR" que podrían ser combinados con una pequeña escala o diario de medidas para evaluar la intensidad y duración de náusea y vómito, e incrementar una pregunta sobre el uso anterior de antieméticos y eventos adversos ${ }^{26}$. El instrumento de evaluación de la fatiga en pacientes con cáncer promueve información cuantitativa para el monitoreo de la condición del paciente y el progreso del tratamiento, la información puede mejorar la comunicación entre el profesional y el paciente, y ser incluida hasta en la decisión del tratamiento que se proponga; el instrumento utilizado fue el "Wu Cancer Fatigue Scale" (WCFS) aplicado en mujeres con cáncer de mama, sin embargo se hace necesaria la validación en pacientes con otros tipos de cáncer, de tratamiento y en otras poblaciones ${ }^{38}$.

La evaluación de la dificultades para la alimentación en pacientes ancianos con demencia fue realizada a través del instrumento "Edinburgh Feeding Avaluation in Dementia" (EdFED) que obtuvo correlación significativa entre las intervenciones de Enfermería (supervisión, ayuda física y nivel de cuidado de enfermería) y los indicadores de dificultad para la alimentación (derramar o dejar comida en el plato después de comer) ${ }^{19}$. El instrumento "Nutrition Screening Tool" (NST) procuró identificar el estado nutricional de pacientes ancianos hospitalizados y mediante éste se pudo documentar esa evaluación, es recomendado para Centros de atención ambulatorios u hospitalarios ${ }^{34}$. La previsión de pérdida de peso y evaluación del apetito puede ser realizada a través de ocho ítems del "Conucil on Nutrition appetite questionnaire" (CNAQ) y dos ítems derivaos del "Simplified Nutritional Appetite Questionnaire" (SNAQ), ambos instrumentos fueron considerados eficientes clínicamente y facilitaron la identificación precoz del riesgo de anorexia por pérdida de peso ${ }^{33}$. La evaluación del amamantamiento en neonatos puede realizarse por el "NOMAS" considerado con propiedades psicométricas consistentes y completo para evaluar tanto la lactancia al seno materno como por biberón ${ }^{27}$.

La evaluación de depresión en ancianos y la importancia de la efectividad en el rastreo de síntomas depresivos en esta población fue mostrada al aplicar el instrumento "GDS Score", los enfermeros detectaron en el $50.1 \%$ de los ancianos síntomas correspondientes ${ }^{28}$. Otro estudio $^{18}$ al aplicar los 5 ítems de la "Geriatric Depression Scale" (GDS) para identificar depresión en ancianos, mostró concordancia significativa con el diagnóstico clínico, el 48.1\% 
de esta población estaba deprimida. El instrumento "ISAR" fue validado con el objetivo de identificar pérdidas funcionales severas y depresión en ancianos, y evaluar los resultados de intervenciones subsecuentes ${ }^{16}$. La evaluación de depresión en pacientes en cuidados paliativos es relevante, y en muchas ocasiones no es considerada en la práctica clínica, sin embargo, puede ser realizada mediante la escala visual ${ }^{35}$.

Para la evaluación de la psicosis fueron utilizados los instrumentos "Positive and Negative Syndrome Scale" (PANSS) e "Routine Assessment of Patients Progress" (RAPP), que pudieron encontrar diferencias en los scores entre grupos con el mismo diagnóstico, demostrando la caracterización del padrón de manifestación de la psicosis; al tener enfocada la importancia de la práctica basada en evidencias, el uso de los instrumentos fue considerado relevante para documentar los resultados clínicos ${ }^{20}$. Mediante los instrumentos "Millon Multiaxial Clinical Inventory" (MMCl-II) y la "Entitlement Scale" (ES) (a Cronbach=.9740) con la finalidad de evaluar rasgos de personalidad, fueron verificados rasgos de agresión, sociopatía e paranoia, con los que fue posible realizar intervenciones terapéuticas basadas en la evidencias del estudio ${ }^{6}$. Otro estudio utilizó para la práctica clínica un instrumento para prever la violencia de pacientes psiquiátricos, denominado como "BVC-VAS", este demostró ser fácil de usar y preciso para la predicción de ataques violentos en servicios de enfermerías psiquiátricas ${ }^{7}$. Este mismo instrumento puede ser aplicado al tener sensibilidad de $64.3 \%$ y especificidad de $93.9 \%{ }^{44}$.

El instrumento "NEECHAM Confusion Scale" fue utilizado para identificar delirio en pacientes ancianos hospitalizados, monitorear el curso de la disfunción cognitiva y la severidad del delirio ${ }^{10}$. En otro estudio, el objetivo fue construir un instrumento llamado "Confusion assessment method for the intensive care unit" (CAM-ICU) para prevenir el delirio en unidades de terapia intensiva por medio de la evaluación de factores estresores físicos, químicos y ambientales a los que los pacientes están expuestos, se encontró un porcentaje de delirio de $48 \%$, dato semejante al de otros estudios en el área ${ }^{17}$.

Las necesidades de cuidado al final de la vida fueron evaluadas por el instrumento "Needs at the End-of-Life Screening Tool" (NEST) con el fin de determinar el curso de las acciones y los resultados deseados por el paciente, pudiendo abordar las necesidades social, existencial, sintomática y terapéutica ${ }^{31}$. La actividad física de los pacientes ancianos institucionalizados fue evaluada mediante la "Continuing Care Activity Measure" (CCAM) donde se pudieron diferenciar los niveles y cambios en la función motora gruesa, estimulando el enfoque en la movilización de esta población, con lo que pudieron reducirse los costos asociados al tiempo de enfermería y hubo una contribución para el bienestar ${ }^{36}$. La incomodidad o disconfort en pacientes con Alzheimer también fue objeto de evaluación mediante la "Discomfort Scale Dementia os Alzheimer Type" (DS-DAT) considerado de fácil uso, rápido, efectivo para ayudar a los profesionales de la salud principalmente de Enfermería ya que interactúan regularmente con los pacientes y por tanto necesitan de instrumentos confiables para evaluar el confort o bienestar ${ }^{43}$.

\section{CONSIDERACIONES FINALES}

Destacamos que hubo un aumento en la producción de publicaciones sobre el tema entre 1999 a 2006. Observamos que la mayoría de los artículos (47.5\%) fueron enfocados a población anciana con instrumentos específicos para el área de Geriatría y Gerontología, que actualmente tiene trascendencia por el envejecimiento de la población a nivel mundial. La disciplina de Enfermería al utilizar instrumentos validados en su práctica pasa a ejercer una asistencia con mayor calidad. No obstante, es evidente que diversos comportamientos y 
manifestaciones clínicas aún carecen de instrumentos validados para su evaluación, y en muchas ocasiones lo existentes son desconocidos por el personal de Enfermería.

\section{REFERENCIAS}

1. Carvalho EC, Bachion MM, Dalri MCB, Jesus CA. Obstáculos para a Implementação do Processo de Enfermagem no Brasil. Rev. Enf. UFPE On Line. 2007; 1(1): 95-99.

2. Conselho Federal de Enfermagem, Resolução COFEN 272/2002. Dispõe sobre Sistematização da Assistência de Enfermagem - SAE. Rio de Janeiro: COFEn; 2002.

3. Carvalho EC, Martins FTM, Dalri MCB, Canini SRMS, Laus AM, Bachiuon MM, Rossi LA. Relações entre a coleta de dados, diagnósticos e prescrições de enfermagem a pacientes adultos de uma unidade de terapia intensiva. Rev Latino-am Enfermagem 2008; 16(4).

4. Webb Christine. Writing for publication: An easy-to-follow guide for any nurse thinking of publishing their work. London: Wiley-Blackweel; 2008.

5. Haines TP; Hill K; Walsh W; Osborne R. Design-related bias in hospital fall risk screening tool predictive accuracy evaluations: systematic review and meta-analysis. J Gerontol A Biol Sci Med Sci 2007; 62(6): 664-72.

6. Kerr NJ. Research validation of misguided entitlement. Int J Psychiatr Nurs Res 2006; 11(2):1269-82.

7. Abderhalden C; Needham I; Miserez B; Almvik R; Dassen T; Haug HJ; Fisher JE. Predicting inpatient violence in acute psychiatric wards using the Broset-ViolenceChecklist: a multicentre prospective cohort study. J Psychiatr Ment Health Nurs 2004; 11(4):422-7.

8. Lee LY; Lee DT; Woo J; Wong EM. Validation of the Chinese version of the satisfaction with the nursing home instrument. J Clin Nurs 2006; 15(12):1574-82.

9. Williams RM; Schmuck G; Allwood S; Sanchez M; Shea R; Wark G. Psychometric evaluation os health work outcome measures for musculoskeletal disorders: a systematic review. J Occup Rehabil 2007; 17(3):504-21.

10. Milisen K; Foreman MD; Hendrickx A; Godderis J; Abraham IL; Broos PL; De Gees S. Pychometric properties of the Flemish translation of the NEECHAM Confusion Scale. BMC Psychiatry 2005; 5:16.

11. Curley MA; Harris SK; Fraser KA; Johnson RA; Arnold JH. State Behavioral Scale: a sedation assessment for infants and young children supported on mechanical ventilation. Pediatr Crit Care Med 2006; 7(2):107-14.

12. Hendrich AL; Bender PS; Nyhuis A. Validation of the Hendric II Fall Risk Model: a large concurrent case/control study of hospitalized patients. Appl Nurs Res 2003; 16(1):9-21.

13. Osborne RH; Hawthorne G; Lew EA; Gray LC. Quality of life assessment in the community-dwelling elderly: validation of the Assessment of Quality of Life (AQoL) Instrument and comparison with the SF-36. J Clin Epidemiol 2003; 56(2):138-47.

14. Lainscak M; Keber I . Validation of self assessment patient Knowledge questionnaire for heart failure patients. Eur J Cardiovas Nurs 2005; 4(4):269-72.

15. Cohen-Mansfield J; Waldhorn R; Werner P; Billing N. Validation of sleep observations in a nursing home. Sleep 1990; 13(6):512-25.

16. Denduki N; McCusker J; Belzile E. The identification of seniors at risk screening tool: further evidence of occurrent and predictive validity. J Am Geriatr Soc 2004; 52(2):290-6.

17. Larsson C; Axell AG; Ersson A. Confusion assessment for the intensive care unit (CAM-ICU):translation, retranslation and validation into Swedish intensive. Acta Anaesthesiol Scand 2007; 51(7):888-92. 
18. Rinaldi P; Mecocci P; Benedetti C; Ercolani S; Bregnocchi M; Menculini G; Catani M; Senin U; Cherubini A. Validation of the five-item geriatric depression scale in elderly subjects in three different settings. J Am Geriatr Soc 2003; 51(5):694-8.

19. Watson R. Measuring feeding difficulty in patients with dementia: replication and validation of the EdFED Scale\#1. J Adv Nurs 1994; 19(5):850-5.

20. Ehman TS; Holliday SG; MacEwan GW; Smith GN. Multidimensional assessment of psychosis: a factor-analytic validation study of the Routine Assessment of Patient Progress. Compr Psychiatry 2001; 42(1):32-8.

21. Delbaere K; Close JC; Menz HB; Cumming RG; Cameron ID; Sambrook PN; March LM; Lord SR. Development and validation of fall risk screening tools for use in residential aged care facilities. Med J Aust 2008;189(4):193-6.

22. Holzemer WL; Henry SB; Nokes KM; Corless IB; Brown MA; Powell-Cope GM; Turner JG; Inouye J. Validation of the Sign and Symptom Check-List for Persons with HIV Disease (SSC-HIV). J Adv Nurs 1999; 30(5):1041-9.

23. Wahle M; Haller S; Spiegel R. Validation of the NOSGER (Nuse's Observation Scale for Geriatric Patients): reability and validity of a caregiver rating instrument. Int Psychogeriatr 1996. 8(4):525-47.

24. Holzemer WL; Henry SB; Stewart A; Janson-Bjerklie S.The HIV quality audit marker (HIV-QAM): an outcome measure for hospitalized AIDS patients. Qual Life Res 1993; 2(2):99-107.

25. Birchley G. Opioid and benzodiapezine withdrawal syndromes in the paediatric intensive care unit: a review of recent literature.Nurs Crit Care 2009; 14(1):26-37.

26. Brearley SG, Clements CV, Molassiotis A. A review of patient self-report tools for chemotherapy-induced nausea and vomiting. Support Care Cancer 2008; 16(11): 1213-29.

27. Howe TH, Lin Kc, Fu CP, Su CT, Hsieh CL. A review os psychometric properties of feeding, assessment tools used in neon ates. J Obstet Gynecol Neonatal Nurs 2008; 37(3):338-49.

28. Thompson P, Lang L, Annells M. A systematic review of the effectiveness of in-home community nurse led interventions for the mental health of older persons. J Clin Nurs 2008; 17(11):1419-27.

29. Miceli DG; Strumpf NE; Reinhard SC; Zanna MT; Fritz E. Current approaches to postfall assessment in nursing homes. J Am Med Dire Assoc 2004; 5(6): 387-94.

30. Gray-Miceli DL; Strumpf NE; Johnson J; Draganescu M; Ratcliffe SJ. Psychometric properties of the Post-Fall Index. Clin Nurs Res 2006; 15 (3): 157-76.

31.Emanuel LL; Alpert HR; Emanuel EE. Concise screening question for clinical assessments of terminal care: the needs near the end-of-life care screening tool. J Palliat Med 2001; 4(4): 465-74.

32. Kohler L; Weyerer S; Schaufele M. Proxy screening tools improve the recognition of dementia in old-age homes: results of a validation study. Age Ageing 2007; 36(5): 54954.

33. Wilson MM; Thomas DR; Rubenstein LZ; Chibnall JT; Anderson S; Baxi A; Dielbold MR; Morley JE. Appetite assessment: simple appetite questionnaire predicts weight loss in community-dwelling adults and nursing home residents. Am J Clin Nutr 2005; 82 (5): 1074-81.

34. Mackintosh MA; Hankey CR. Reability of a nutrition screening tool for use in elderly day hospitals. J Hum Nutr Diet 2001; 14(2): 129-36.

35. Lees N; Lloyd-Williams M. Assessing depression in palliative care patients using the visual analogue scale: a pilot study. Eur J Cancer Care (Engl) 1999; 8 (4): 220-3.

36. Huijbregts MP; Teare GF; McCullough C; Kay TM; Streiner D; Wong SK; McEwen SE; Otten I. Standardization of the continuing care activity measure: a multicentre study to 
acess reliability, validity, and ability to measure change. Phys Ther 2009; 89 (6):54655.

37. Suriadi; Sanada H; Sugama J; Thigpen B; Subuh M. Development of a new risk assessment scale for predicting pressure ulcers Iná na intensive care unit. Nurs Crit Care 2008; 13 (1): 34-43.

38. Wu HS, Wyrwich KW; Mc Sweeney M. Assesseing in persons with cancer: further validation of the Wu Cancer Fatigue Scale. J Pain Symptom Manage 2006; 32(3):25565.

39. Lam CL; Chan WC; Mok CC; Li SW; Lam LC. Validation of the Chinese Challenging Behaviour Scale: clinical correlates of challenging behaviours in nursing home residents with dementia. Int J Geriatr Psychiatry 2006; 21(8):792-9.

40. Pautex S; Herrmann F; Le Lous P; Fabjan M; Michel JP; Gold G. Feasibility and reability of four pain self-assessment scales and correlations with an observational rating scale in hospitalized elderly demented patients. J Gerontol A Biol Sci Med Sci 2005; 60(4): 524-9.

41. McNair C; Ballantyne M; Dione K; Stephens D; Stevens B. Postoperative pain assessment in the neonatal intensive care unit. Arch Dis Child Fetal Neonatal Ed 2004; 89 (6): F537-41.

42. Beck SL; Schwartz AL; Towsley G; Dudley W; Barsevick A. Psychometric evaluation of the Pittsburg Sleep Quality Index in cancer patients. J Pain Symptom Manage 2004; 27(2):140-8.

43. Dello Russo C; Giulio PD; Brunelli C; Dimonte V; Villani D; Renga G; Toscani F. Validation of the Italian version of the Disconfort Scale - Dementia of Alzheimer Type. Journal of Advanced Nursing. 2008; 64(3):298-303.

44. Abderhalen C; Needham I; Miserez B; Almvik R; Dassen T; Haug H-J; Fischer JE. Predicting inpatient violence in acute psychiatric wards using the Broset-ViolenceChecklist: a multicentre prospective cohort study. Journal of Psychiatric and Mental Health Nursing 2004;11: 422-27. 